\title{
Development of the TanDEM-X Calibration Concept: Analysis of Systematic Errors
}

\author{
Jaime Hueso González, Markus Bachmann, Gerhard Krieger, Member, IEEE, and Hauke Fiedler
}

\begin{abstract}
The TanDEM-X mission, result of the partnership between the German Aerospace Center (DLR) and Astrium GmbH, opens a new era in spaceborne radar remote sensing. The first bistatic satellite synthetic aperture radar mission is formed by flying TanDEM-X and TerraSAR-X in a closely controlled helix formation. The primary mission goal is the derivation of a high-precision global digital elevation model (DEM) according to High-Resolution Terrain Information (HRTI) level 3 accuracy. The finite precision of the baseline knowledge and uncompensated radar instrument drifts introduce errors that may compromise the height accuracy requirements. By means of a DEM calibration, which uses absolute height references, and the information provided by adjacent interferogram overlaps, these height errors can be minimized. This paper summarizes the exhaustive studies of the nature of the residual-error sources that have been carried out during the development of the DEM calibration concept. Models for these errors are set up and simulations of the resulting DEM height error for different scenarios provide the basis for the development of a successful DEM calibration strategy for the TanDEM-X mission.
\end{abstract}

Index Terms-Baseline, digital elevation model (DEM) calibration, interferometry, phase errors, synthetic aperture radar (SAR), TanDEM-X.

\section{INTRODUCTION}

$\mathbf{T}$ HE TanDEM-X satellite (TDX) will be launched in the last quarter of 2009 to join its twin satellite TerraSAR-X (TSX), in orbit since June 2007, and to form the first bistatic single-pass synthetic aperture radar (SAR) satellite formation [1]. The satellites, originally designed for standalone monostatic operations, have been upgraded to be also capable of performing bistatic acquisitions, thus enabling the derivation of DEMs from SAR interferometry (InSAR [2]) during three years of combined operations. The TanDEM-X mission [1] represents a new generation of complex spaceborne SAR systems, and it has the challenge to deliver a global DEM with HRTI-3-like height accuracy (Table I and [3]) within four years after launch.

The only comparable project was the Shuttle Radar Topography Mission (SRTM/X-SAR) [4], which mapped three dimensionally $80 \%$ of the Earth's landmass in 2000.

Manuscript received February 27, 2009; revised July 10, 2009, August 24, 2009, and October 8, 2009. First published December 15, 2009; current version published January 20, 2010. This work was supported in part by the German Federal Ministry for Economics and Technology (Förderkennzeichen 50 EE 0601).

The authors are with the Microwaves and Radar Institute, German Aerospace Center (DLR), 82234 Wessling, Germany (e-mail: jaime.hueso@dlr.de; markus.bachmann@dlr.de; gerhard.krieger@dlr.de; hauke.fiedler@dlr.de).

Color versions of one or more of the figures in this paper are available online at http://ieeexplore.iee.org.

Digital Object Identifier 10.1109/TGRS.2009.2034980
TABLE I

TANDEM-X DEM SPECIFICATIONS

\begin{tabular}{|l|l|l|}
\hline Requirement & Specification & HRTI-3 \\
\hline Absolute vertical accuracy (global) & $90 \%$ linear error & $10 \mathrm{~m}$ \\
\hline $\begin{array}{l}\text { Relative vertical accuracy } \\
(\text { within } 100 \mathrm{~km} \times 100 \mathrm{~km})\end{array}$ & $\begin{array}{l}90 \% \text { linear point-to- } \\
\text { point error }\end{array}$ & $\begin{array}{l}2 \mathrm{~m}(\text { slope }<20 \%) \\
\text { (slope }>20 \%)\end{array}$ \\
\hline Horizontal resolution & Independent posting & $12 \mathrm{~m}$ \\
\hline
\end{tabular}

The SRTM's InSAR-derived DEM achieved a relative height accuracy on the order of $10 \mathrm{~m}$ at 1 -arcsec resolution. The corresponding TanDEM-X mission, with its relative height accuracy requirement of $2 \mathrm{~m}$ (cf. Table I), sets therefore, a new standard in DEM quality and coverage.

As already shown by the SRTM/X-SAR DEM calibration experience [5], the correction of systematic height errors in the DEM is necessary to achieve the specified accuracies. The TanDEM-X DEM calibration concept has also to consider the specifics of having two independent SAR instruments on two satellites forming a single-pass bistatic InSAR configuration, like different instrument phase drifts or baseline errors caused by the limitations of the GPS measurements onboard the satellites. The approach of using sea-level heights (ocean-land-ocean acquisitions) as applied in [5] is not feasible for the TanDEM-X mission, as its operational helix configurations, with along-track baselines typically longer than $200 \mathrm{~m}$, cause temporal decorrelation and phase shifts over moving water surfaces [1]. Furthermore, TanDEM-X mission data acquisitions or "datatakes" (DTs) are too short for this kind of calibration measurements, as their length is limited (normal: 50-200 s, maximal: $300 \mathrm{~s}$ ) due to power/thermal and data storage/downlink constraints.

Nevertheless, the expected performance losses, the limited signal-to-noise ratio of the SAR systems, and other noiselike sources cause phase variances that lead to saturate most of the TanDEM-X DEM relative height error budget of $2 \mathrm{~m}$. Therefore, a new robust DEM calibration strategy that minimizes the systematic height errors is essential.

The main motivation of this paper is to describe the development of this DEM calibration concept, capable of fulfilling its demanding requirements. Section II provides a brief review of the fundamental relations between the interferometric phase and the terrain height, as well as it presents some key aspects of the TanDEM-X mission. Section III describes the TanDEM-X mission-specific error sources that could affect the accuracy of the InSAR-derived DEM (or terrain height). Special focus is given to the baseline determination errors in Section IV, as they may range between $1-3 \mathrm{~mm}$ and are the main systematic height error contribution. Based on the error analysis presented in 


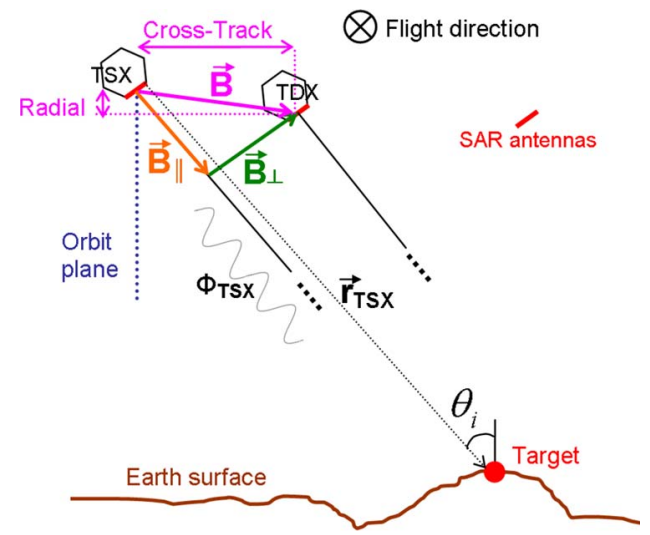

Fig. 1. SAR interferometry imaging geometry for the TanDEM-X mission.

Sections III and IV, a suitable correction model is derived, which is discussed in Section V along with some simulation results. The paper concludes in Section VI with some considerations about the expected quality of the final TanDEM-X global DEM.

\section{INSAR AND TANDEM-X FUNDAMENTALS}

\section{A. TanDEM-X Interferometric Phase and Height Errors}

The SAR interferometry imaging geometry for the TanDEM-X mission is shown in Fig. 1.

Bistatic interferometric SAR data acquisitions for DEM generation consist of one satellite transmitting radar pulses (active), while both satellites receive simultaneously the radar echoes from the ground. The satellite in receive-only mode is the passive satellite. The interferogram is computed from the phase difference between the complex monostatic and bistatic SAR images. In TanDEM-X mission DTs, either TSX or TDX can be selected as the active satellite depending on the relative position between them (one satellite should not directly illuminate the other one) and on power-consumption considerations.

The relative position or baseline $B$ is defined as the vector that links the two SAR antenna phase centers. Assuming zero along-track displacement and following the notation of Fig. 1, the main relation between the bistatic interferometric phase and $B$ is [2]

$$
\phi \approx-\frac{2 \pi}{\lambda}\langle\hat{r}, \vec{B}\rangle
$$

where $\lambda$ is the SAR signal wavelength and $\hat{r}$ is the slant range unitary vector from the emitting satellite to the ground target. The approximation in (1) is justified by the fact that $|B| \ll|r|$.

Fig. 1 also shows the decompositions of the baseline vector $B_{\|}$and $B_{\perp} . B_{\|}$is the projection of $B$ parallel to the line-ofsight (LOS) direction, whereas $B_{\perp}$ (also called effective baseline) is the corresponding projection perpendicular to the LOS. Subtracting from the interferometric phase, the contribution of the flat Earth, the remaining phase history represents the height changes over the Earth surface.

The height of ambiguity $h_{\mathrm{amb}}$ is a key InSAR performance parameter providing the information on the terrain height that can be unambiguously resolved within the $2 \pi$ definition range of the interferometric phase

$$
h_{\mathrm{amb}}=\frac{\lambda r \sin \left(\theta_{i}\right)}{B_{\perp}}
$$

where $\theta_{i}$ is the incidence angle. This equation points out that $B_{\perp}$ controls the phase-to-height conversion and determines the height resolution of the system.

Instrument phase drifts and noise contributions affect the quality of the interferogram in the following way [1], [6].

1) They cause height errors, as can be directly derived from (1) and (2)

$$
h_{\mathrm{err}}=\frac{h_{\mathrm{amb}}}{2 \pi} \cdot \phi_{\mathrm{err}} .
$$

Baseline errors have different impacts depending on their orientation with respect to the SAR LOS [1], [6].

2) Along-track baseline errors are resolved during processing and are therefore uncritical.

3) Baseline errors perpendicular to the $\operatorname{LOS}\left(B_{\perp_{\text {err }}}\right)$ cause a bias in the phase-to-height scaling, which, in the TanDEM-X mission, practically results in errors on the order of few centimeters.

4) Baseline errors parallel to the $\operatorname{LOS}\left(B_{\| \text {err }}\right)$ cause a vertical displacement of the DEM

$$
h_{\mathrm{err}}=\frac{h_{\mathrm{amb}}}{\lambda} \cdot B_{\| \mathrm{err}} .
$$

As $h_{\mathrm{amb}}$ varies with $\theta_{i}$, the vertical displacement causes a tilt in the ground range of the DEM given by

$$
\varphi_{\mathrm{tilt}}=\frac{h_{\mathrm{err}}}{\Delta s}=\frac{B_{\| \mathrm{err}}}{B_{\perp}}
$$

where $\Delta s$ is the ground range distance from a selected reference point.

The $h_{\mathrm{amb}}$ of the system is constantly changing during flight (e.g., from 39 to $41 \mathrm{~m}$ for a typical M-C1 mission phase [1] and normal DT length), as the baseline follows a cyclical helix configuration (like Fig. 2). As height errors motivated both by baseline and phase errors are dependent on $h_{\mathrm{amb}}$, their temporal evolution is modified with the changes of the baseline configuration and the incidence angle.

This implies a small change of the height error in azimuth in any acquired interferometric DT, even if the baseline error would be constant. This effect introduces certain nonlinearities in the phase-to-height conversion of the errors, which complicates the design of DEM correction functions.

\section{B. Mission and Acquisition Plan}

A fine adjustment of the orbit parameters of both satellites (horizontal orbital displacement by different ascending nodes with a vertical separation by different eccentricity vectors) results in a closely controlled helix formation with typical crosstrack baselines between 250 and $500 \mathrm{~m}$ (Fig. 2 and [1]). 
The parameters of the helix configuration are modified during the mission in order to enable an interferometric mapping of the complete Earth surface with a stable $h_{\mathrm{amb}}$.

The best conditions for interferometric SAR image acquisitions with respect to DEM generation are the orbit instants with maximal effective baseline (black arrows in Fig. 2), and therefore, minimal and slowly changing $h_{\mathrm{amb}}$. As a result of this strategy, the Northern Hemisphere will be mapped at least twice with scaled baselines using ascending orbits and, analogously, the Southern Hemisphere with descending orbits.

\section{DEM Processing and Calibration}

The DEM-generation chain of the TanDEM-X system is triggered when the baseline product, based on GPS navigation data recorded by both satellites, is available.

The first stage, the interferometric TanDEM-X processor (ITP), derives the SAR images, georeferences them, and calculates the raw DEMs. The georeferencing is supported by the external calibration of the SAR system, which takes place during the satellite-commissioning phase. The ITP implements multibaseline phase-unwrapping algorithms [7] and techniques like the delta-k interferometry [8] in order to minimize phaseunwrapping errors. It is foreseen to acquire data sets over difficult terrain (like mountainous areas) with three or more different effective baselines to improve the robustness of the method.

Moreover, within the ITP, other corrections take place, like the compensation of the SAR antenna phase patterns and the correction of the SAR instrument phase drifts by applying the internal calibration replicas. As each satellite has its own local oscillator, relative phase drifts are monitored via a dedicated synchronization link, through which special sync pulses are periodically exchanged between the twin satellites during a TanDEM-X mission DT. The sync pulses are stored in the same way as imaging pulses and are then evaluated on ground by the ITP to derive a compensation phase to apply to the interferogram.

However, the corrected raw DEMs from the ITP may still contain residual height errors. The DEM calibration concept is the strategy to compensate them. The concept is implemented by the Mosaicking and Calibration Processor (MCP) [9], which is in charge of delivering the final TanDEM-X global DEM at the end of the mission, once all raw DEMs are available. The DEM calibration concept also defines the selection of suitable height references and gives inputs to the data acquisition plan.

\section{RESIDUAL-ERROR SOURCES}

The raw DEMs delivered by the processor are still affected by several error sources like instrument-phase noise and coherence losses that are dependent e.g., on the SAR antenna radiation pattern and the Earth surface characteristics. In addition, the SAR instrument calibration and the oscillatorsynchronization procedures do have residual errors. This section includes a classification of the different residual-error contributions depending on their nature, as well as an analysis of the instrument-phase errors. A summary of the systematic instrument errors is provided in Table II.
TABLE II

SAR Instrument-Related Residual Systematic Phase ERrors

\begin{tabular}{|l|c|c|c|}
\hline Contribution & Nature & Phase Error & $\begin{array}{l}\text { Height Error } \\
\left(\text { typical } h_{a m b}\right)\end{array}$ \\
\hline $\begin{array}{l}\text { Internal calibration } \\
\text { - TempComp }\end{array}$ & linear & $1 \% / 100 \mathrm{~s}$ & $\begin{array}{c}0.1 \mathrm{~m}(1 \sigma) \\
/ 100 \mathrm{~s}\end{array}$ \\
\hline $\begin{array}{l}\text { Bistatic mode - } \\
\text { Cal Network }\end{array}$ & $\begin{array}{c}\text { temperature } \\
\text { dependent }\end{array}$ & $\begin{array}{c}\text { cancelled out } \\
\text { OGC polynom. }\end{array}$ & - \\
\hline Sync Link - LAAs & $\begin{array}{c}\text { negative } \\
\text { exponential }\end{array}$ & $\begin{array}{c}0.5^{\circ} \\
\text { after } 100 \mathrm{~s}\end{array}$ & $\begin{array}{c}0.05 \mathrm{~m}(1 \sigma) \\
\text { after } 100 \mathrm{~s} ; \\
\sim 0.3 \mathrm{~m} \text { long DTs }\end{array}$ \\
\hline
\end{tabular}

\section{A. Classification}

The main sources of the arising height errors in the DEM can be classified into three groups (cf. Fig. 3):

1) interferometric phase errors that have a fast/"noiselike" variation rate;

2) residual drifts or offsets in the phase determination within the radar instruments;

3) inaccuracies in the baseline determination.

The aforementioned error contributions can be studied from the perspective of their variation rate within the typical DT length. Errors due to limited signal-to-noise ratio, volume decorrelation, oscillator-phase noise, random baseline errors and noiselike synchronization inaccuracies are placed among the fast noiselike errors. White-noise components can be mitigated by using interferometric looks [6]. However, colored noise (typically, with components higher than $1 \mathrm{~Hz}$ ) does not necessarily decrease by means of these techniques.

On the other hand, baseline inaccuracies and systematic instrument drifts can be mainly classified as systematic errors, which range depending on their nature between medium-fast to very slow changing errors, with the baseline errors having the largest impact on the InSAR height accuracy. These errors can be easily corrected for, as the number of required height references and the complexity of the correction functions decrease with the error-variation rates.

Due to the close formation of both satellites, external perturbations affect both satellites equally. As the along-track distance between the satellites is typically less than $1 \mathrm{~km}$, both satellites have very similar signal paths through the atmosphere, which means almost identical atmospheric effects that do not affect the interferometric performance. The same applies for solar wind pressure and drag.

\section{B. Noiselike Phase Errors}

Most of the noiselike errors that affect the TanDEM-X DEMs are motivated by performance losses, which may be due to volume decorrelation in forested areas or losses in mountainous terrain. Other sources originate in the SAR instrument, like analog-to-digital converter quantization errors and limited signal-to-noise ratio (SNR). The instrument frequency is controlled by an ultrastable oscillator (USO) [11], but has nevertheless a characteristic phase noise profile. Finally, the finite SNR of the synchronization link, the limited sync-pulse exchange frequency, and potential multipath effects can cause inaccuracies in the phase correction of the interferogram [1], [12].

All these sources have been analyzed, and their contribution on the DEM height has been quantified [13]. Simulations show 


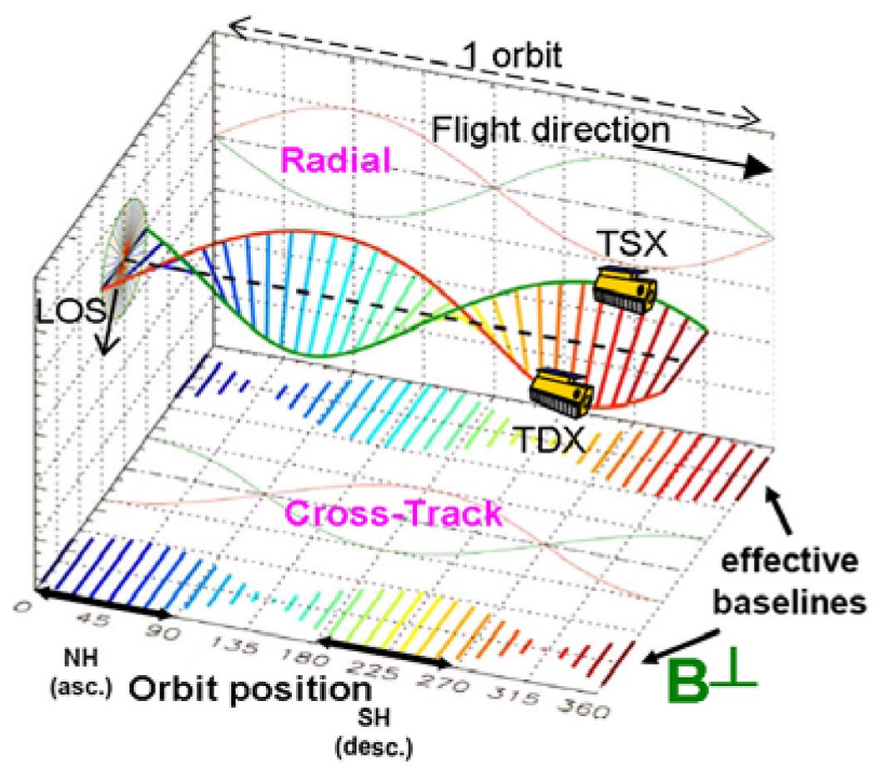

Fig. 2. TanDEM-X mission helix formation and baselines over one orbit.

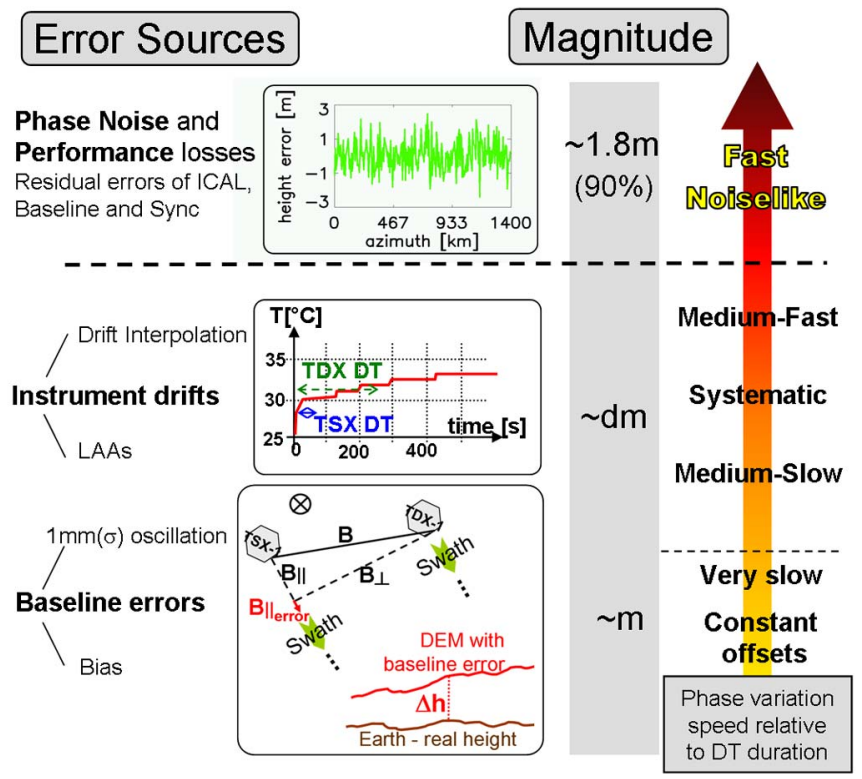

Fig. 3. Nature and impact of systematic and noiselike errors on the DEM. Normal DT duration of 50-200 s assumed.

that, for worst case scenarios, the height error due to the overall noiselike error components is around $1.8 \mathrm{~m}(90 \%$ confidence level) after interferometric multilooking [13]. This leaves little margin for accommodating other error contributions, as the specification for the total relative height error budget is $2 \mathrm{~m}$ (90\%). Specifically, it means that the remaining margin for systematic error correction is $0.53 \mathrm{~m}(1 \sigma)$. This is the accuracy goal for the DEM calibration.

\section{Residual Instrument Phase Drifts}

1) Signal Paths in the SAR Instrument: The radar pulses' phase evolution due to propagation within each SAR instrument does not remain constant over time. Phase distortions occur

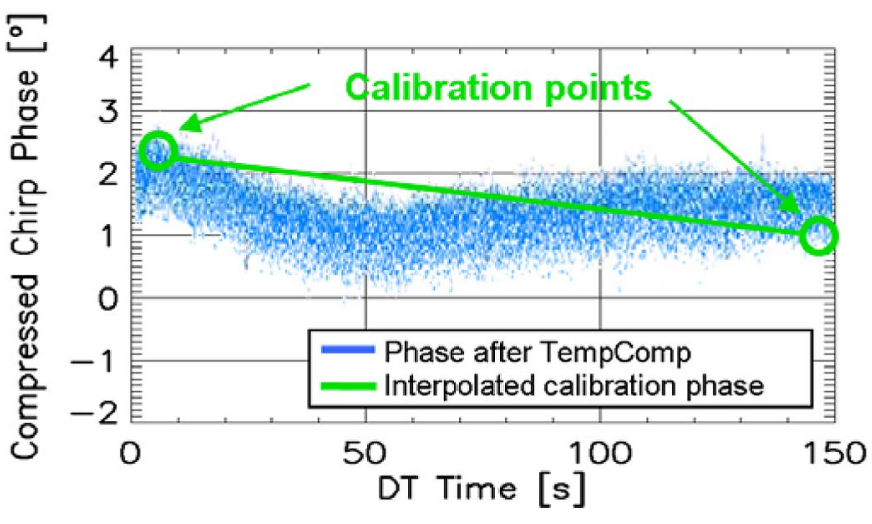

Fig. 4. Residual internal calibration error.

on the transmit and receive paths due to temperature effects not only mainly in the amplifiers and transmit/receive modules (TRMs) but also in passive devices like power dividers and transmission lines. These effects are monitored with the internal calibration loop and compensated during processing. Differential phase drifts between both satellites, as both instruments have independent USOs and temperature profiles over time, are covered by the synchronization link.

2) Internal Calibration: The internal calibration is suited for correcting slow phase and amplitude drifts of the instrument. The Temperature Compensation Mode (TempComp) [16] is a temperature control strategy during satellite operations that automatically measures and adjusts the TRM amplifiers of the antenna electronics in order to stabilize the working point of the instrument under temperature variations. One of the consequences of using this mode is that a simple linear interpolation can be applied to correct for the instrument drift along a DT (cf. Fig. 4). The remaining errors can be described with a systematic linear component, with typical slopes of less than $1^{\circ}$ after $100 \mathrm{~s}$ and a random component of $0.15^{\circ}(1 \sigma)$.

This results in a systematic height error component of less than $0.1 \mathrm{~m}(1 \sigma)$ for typical height of ambiguities. In the absence of an internal calibration mechanism, the TempComp mode would still keep the instrument relatively stable $\sim 5^{\circ}(1 \sigma)$, causing height errors of around $0.5 \mathrm{~m}$.

3) Bistatic Internal Calibration Approach: The calibration network of the instrument does not have an effect on the radar pulses, but it modifies the calibration pulses that are used to generate the replica of the chirp signal, which is applied to compensate the instrument behavior in the internal calibration [16]. Therefore, changes in temperature that perturb the amplitude and phase behavior of the calibration loop introduce errors in the internal calibration and could have a direct effect on the interferometric accuracy of the system. However, the elements of the calibration chain, which are mainly passive components, have been properly characterized on the ground by means of simple polynomial functions (cf. Fig. 5).

For the TerraSAR-X mission regular stand-alone image acquisition mode, a single amplitude correction value is applied to each DT to compensate the calibration-loop effect. These TerraSAR-X mission acquisitions are usually shorter (around $15 \mathrm{~s})$ than the ones planned for the interferometric SAR image acquisition (50-200 s) during the TanDEM-X mission. 


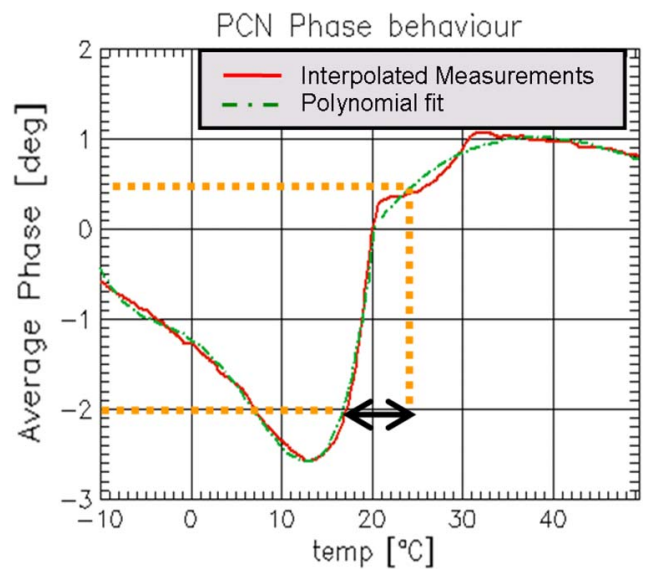

Fig. 5. Example for the phase behavior of the TSX Panel Calibration Network. Black arrow shows typical increment of temperature in TanDEM-X DTs. Source: Astrium Friedrichshafen.

Therefore, the phase effect of the calibration loop is neglected for TerraSAR-X mission DTs.

For the Tan-DEM-X mission, the approach is different: polynomials for both amplitude and phase are used to compensate the effect of temperature changes on the calibration loop. The order of the polynomials is typically five or less. However, in some cases, two polynomials are required to cover the entire temperature range between $-10{ }^{\circ} \mathrm{C}$ and $+50{ }^{\circ} \mathrm{C}$.

As an extreme example, Fig. 5 shows the phase behavior of a critical element of the calibration network. Typical temperature changes in DTs up to $200 \mathrm{~s}$ are close to $10{ }^{\circ} \mathrm{C}$ (the calibration network does not heat up as much as the front end, as it has no active element). In extreme cases, this would imply relatively fast-changing phase errors of around $2.5^{\circ}$, resulting in a relative DEM height error of about $0.25 \mathrm{~m}$. This height error is not dramatic, but its fast-changing nature would complicate its correction in the DEM calibration. It has to be noted that all experiments performed with the TSX satellite to simulate TanDEM-X mission-like DTs have shown smaller calibration network phase errors than the aforementioned. This is due to the fact that the contributions of the different elements of the network tend to slightly compensate each other when considering the total phase effect over temperature. As a conservative strategy, every time a calibration pulse sequence is generated, the correction polynomials are applied, canceling out this type of instrument-phase errors.

4) Synchronization Link: The exchange of special synchronization pulses between the satellites provides coherence to the bistatic system, but there are some residual drifts remaining. When processing the phase difference, all reciprocal components cancel out, and only nonreciprocal components, like amplifiers, produce a residual phase error. As the loop is not covered by the internal calibration correction, temperature drifts in the leaf amplifiers (LAAs) have to be corrected for. This is done using the housekeeping (HK) data from the temperature sensors on these amplifiers. These sensors have a finite accuracy and resolution. On ground, the temperature HK data are compared with the LAAs characterization curves and transformed into phase curves. The rough quantization of the temperature samples causes the obtained phase curves to differ

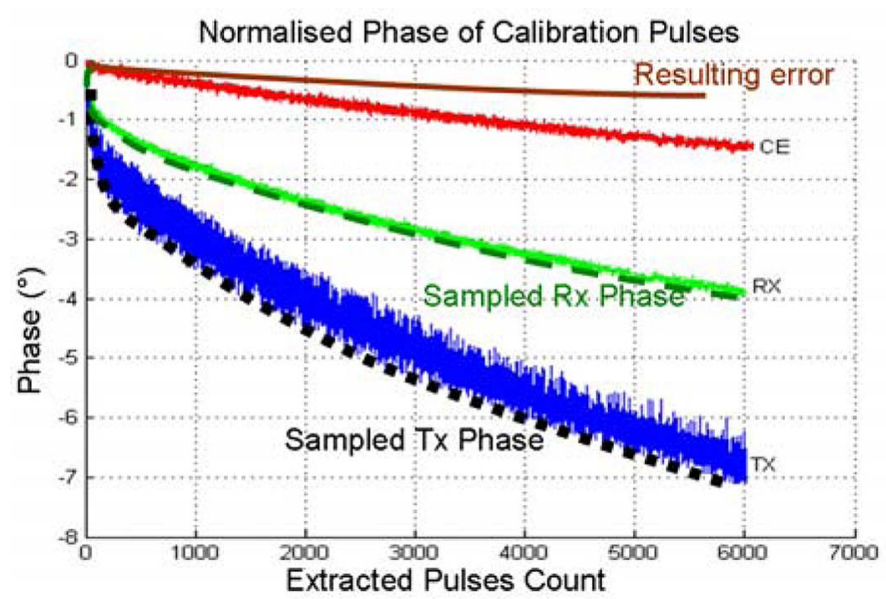

Fig. 6. Example LAA phase evolution, obtained from the internal transmission, reception, and central electronic calibration pulses, and faulty sampling.

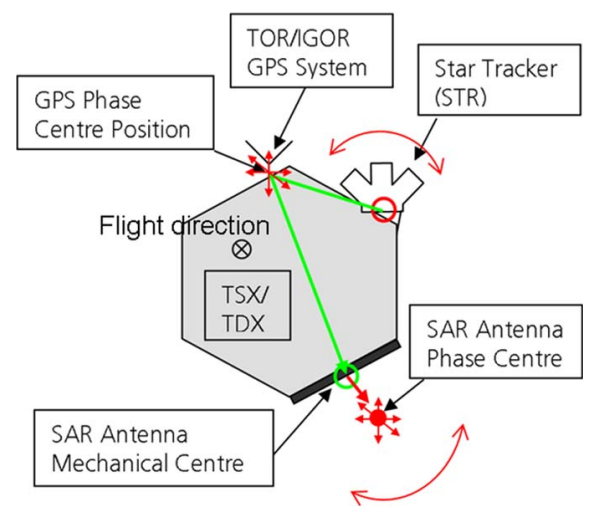

Fig. 7. Scheme of the location of the navigation and attitude determination components in the TSX and TDX payload. Cross section of the satellite.

from the real phase drifts, as shown in the comparison of Fig. 6 based on TSX measurements before launch.

Assuming a realistic temperature gradient of $7{ }^{\circ} \mathrm{C}$ over a DT of maximal length $(300 \mathrm{~s})$, it corresponds to about $7^{\circ}$ phase drift, and the phase estimate is faulty by about $\pm 0.5^{\circ}$, as exemplarily shown in the "resulting error" curve of Fig. 6. The phase error is modeled as a negative exponential function that grows to a value of $0.5^{\circ}(1 \sigma)$ in the first $100 \mathrm{~s}$. The analysis of Fig. 6 shows a mean height error of less than $0.3 \mathrm{~m}(1 \sigma)$ for the whole DT length.

\section{BASELINE DETERMINATION ERRORS}

The generation of accurate InSAR-derived DEMs requires a precise knowledge of the satellite baseline. In the case of the TanDEM-X mission, the accuracy requirement for the baseline determination has been set to $1 \mathrm{~mm}$ in order to minimize the relative height error.

The baseline determination depends principally on the estimation of three parameters, which are the relative satellite orbit position measured with GPS, the spacecraft attitude determined with Star Trackers and the location of the SAR antenna phase center (Fig. 7 shows the distribution of these devices on the satellite payload). 


\section{A. Attitude Determination}

The Star Trackers onboard TSX and TDX are three accurate digital cameras that use a star map to determine their actual position and orientation in space. Due to disturbances like sun or moon light incidence, distortions of the optics, etc., the attitude determination accuracy has a slowly varying bias of $\pm 0.005^{\circ}$ in the yaw, pitch, and roll components plus a $0.003^{\circ}$ sigma random error. A satellite rotation mainly affects the perpendicular baseline in the acquisition geometry and only slightly the parallel one. The resulting bias transforms to a baseline LOS error of less than $0.1 \mathrm{~mm}$, which has almost no impact on the DEM and, therefore, can be neglected.

\section{B. SAR Antenna Phase Center}

The phase center of the SAR antenna describes the variation or displacement of the phase curve within the coverage region against a defined origin, here the origin of the antenna coordinate system. This phase curve depends on the look angle and hence, is different for each beam. The antenna phase patterns have been measured on the ground with an accuracy of better than $3^{\circ}$ in phase. This deviation from the real pattern remains relatively constant over time. The characterized phase curves are applied during processing to compensate the phase patterns in the SAR images. As the antennas and, particularly, their waveguides are manufactured with identical processes for both satellites, and the same beams have to be commanded for both SAR antennas during single-pass bistatic acquisitions, the potential SAR phase center deviations should equally affect the mono- and the bistatic path. Therefore, their influence in the actual baseline is supposed to cancel out, not affecting the DEM quality.

\section{GPS Position Measurements}

The orbit position is computed by the onboard GPS navigation receivers, called Tracking Occultation and Ranging/ Integrated GPS Occultation Receiver (TOR/IGOR) system. The verification on TSX proved that an absolute accuracy of $5 \mathrm{~cm}(1 \sigma)$ is achieved with the TOR/IGOR system fulfilling the requirements.

This accuracy is more than sufficient for the absolutepositioning accuracy. An error in the absolute position transforms directly into a height or position error of the measured target point. It would introduce a relatively constant height error on the order of $5 \mathrm{~cm}$, thus, only affecting the absolute height accuracy requirement of $10 \mathrm{~m}$.

It is possible, however, to track the relative changes between both satellites (baseline) with a much higher accuracy. This is performed through processing the navigation information derived from double differential GPS (DDGPS) carrier phase measurements between both satellites [14] and applying a Kalman-filter method to the data. The use of the differential information even eliminates ionospheric errors and other characteristic GPS perturbations. To further improve the baseline determination accuracy, the exact GPS antenna phase center, which changes with the GPS-signal incidence angle, has been characterized. The effect is then corrected for when generating the baseline product.

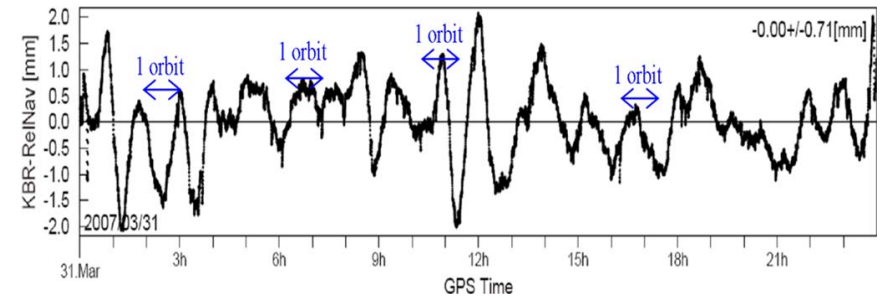

Fig. 8. One-day comparison between Ka and DDGPS baselines by GRACE. Orbit period $\sim 90 \mathrm{~min}$. Standard deviation of the error is $0.71 \mathrm{~mm}<1 \mathrm{~mm}$. Source: Wermuth/Montenbruck (GSOC-DLR).

The resulting baseline determination accuracy is expected to be on the order of millimeters. This assumption is based on the performance of the DDGPS method in similar missions like GRACE [15]. The comparison is possible as GRACE is also a two-satellite constellation with similar orbit period, GPS receivers, and baseline determination methods (DDGPS relative navigation) as the TanDEM-X constellation. The baseline error in the GRACE formation was estimated by comparing the GPSprocessed data with the highly accurate measurements from a dedicated onboard Ka-band link. Fig. 8 shows that the error can be modeled by a low-frequency stochastic process that has a dominant periodical component at around one orbital revolution. The amplitude of the error is of less than $1 \mathrm{~mm}(1 \sigma)$.

This means that the main component of the baseline error is changing "slowly" over time compared with the TanDEM-X mission maximal DT length. The results in TanDEM-X could be even better than in GRACE as the baseline length is much shorter and the GPS receivers have a slightly better performance. This agrees with the baseline accuracy-mission requirements of $1 \mathrm{~mm}$. Nevertheless, the GPS baseline solution accuracy is the main error source for the baseline determination. All other error sources described in Section IV are much smaller.

\section{Baseline Bias and Its Characterization}

Concerning the baseline determination accuracy, it is necessary to point out that the GRACE results do not prove that the 1-mm baseline accuracy is reached in absolute terms. The TanDEM-X DEM calibration has a cautious approach and considers the possibility of having a small offset in the baseline product on the order of 1 to $9 \mathrm{~mm}$. Such an offset could be due to small measurement errors in the location of the baselinereference points in the satellite payload, or small systematic errors of the DDGPS measurements, or other unknown reasons, although the experience in GRACE suggests that it should be almost constant over time (cf. Fig. 9).

An obvious indication for a baseline bias is a significant absolute height error of several meters in the raw DEM, which may range from three to tenths of meters depending on the magnitude of the bias and the $h_{\mathrm{amb}}$. As it has a constant nature, it can be easily corrected for with absolute height references, but there are other side effects.

Up to now, only the effect of baseline and phase errors on the height has been described. In fact, phase and baseline errors parallel to the LOS cause a rotation of the DEM around the flight trajectory, so the height error is always linked to a ground range displacement $g r_{\text {err }}$ (cf. Fig. 10 and [6]). However, its value is small $\left(<4 \mathrm{~m}\right.$ for typical incidence angles and $\left.h_{\mathrm{amb}}\right)$ 
and negligible for the TanDEM-X mission requirements if the baseline offset is below $2 \mathrm{~mm}$.

The eventual presence of a bias on the order of several millimeters in the baseline product invalidates this approximation.

The posting of the TanDEM-X raw DEMs is 6 and $12 \mathrm{~m}$ in the final product, respectively. Ground range displacements have to be considered if their value is on the order of the raw DEM posting or higher, as the DEM might be shifted by one or more pixels. If two adjacent DTs have ground range displacements in opposite directions, the heights in the overlapping regions are compared in different locations, which can cause considerable height errors, particularly in mountainous regions.

As shown in (4), the main contribution to the height error is caused by the baseline error in LOS. In the presence of a baseline bias in LOS larger than $2 \mathrm{~mm}$, it is justified to assume that this is the only significant source of the systematic height error in a DEM.

Following this philosophy, a simple correction method for the ground range displacement has been proposed, as well as a characterization strategy for the baseline bias. The baseline offset in LOS can be estimated through interferometry by calculating the height error of an InSAR-derived DEM over a region with a known accurate reference DEM, obtaining an average height error value and applying (4) to derive the unknown $B_{\| \text {err }}$. If the assumption is taken that the ground range displacement is mainly motivated by the baseline LOS error, it can be expressed as

$$
g r_{\mathrm{err}}=\frac{r \cos \left(\theta_{i}\right)}{B_{\perp}} \cdot B_{\| \mathrm{err}} .
$$

As an example, baseline LOS offsets of around $3 \mathrm{~mm}$ can cause shifts of more than $4 \mathrm{~m}$ in ground range direction. If adjacent DTs would be displaced in opposite directions, the relative displacement would be $8 \mathrm{~m}$, and the height averaging in the overlapping regions would cause considerable height errors.

An obvious solution can be identified after applying (4) to (6)

$$
g r_{\mathrm{err}}=\frac{h_{\mathrm{err}}}{\tan \left(\theta_{i}\right)} .
$$

Hence, the ground range displacement is directly proportional to the height error and can be characterized and corrected for in the same way as the height error. Even simple corrections with average height error values and single ground range displacement correction values for the whole DT offer satisfactory results.

This approach has been proposed to characterize the LOS baseline bias during the TanDEM-X commissioning phase. In order to avoid the coupling of ground range displacement with height errors, the characterization is only performed in selected flat areas over the Earth. In this way, the ground range displacement does not add height errors to the estimation, and no iterative height-error/ground-range displacement estimation is needed.

Once this is done, a calibrated baseline can be generated, and the ITP is then able to deliver raw DEMs with much less rotation (height and ground range displacement). Assuming the baseline bias is properly corrected, the calibration efforts can then focus on the residual height error.

\section{E. Model for the Overall Baseline Error}

The most critical baseline error is $B_{\| \text {err }}$, and the height errors are proportional to it. As an example, a typical $h_{\mathrm{amb}}$ of $35 \mathrm{~m}$ and a $B_{\| \text {err }}$ of $2 \mathrm{~mm}$ leads to a height error of $2.2 \mathrm{~m}$ in the DEM. As neighboring DTs probably have baseline errors with different signs, this can affect the relative height accuracy of the system, which is more critical than the absolute one. Thus, such errors violate the relative height requirement stated in Section III-B.

The overall baseline error is modeled as a sinusoidal function with periodicity close to orbit duration, amplitude of $2 \mathrm{~mm}(1 \sigma)$, and arbitrary initial phase for each DT in all 3-D coordinates, which represents a worst case for all sources. The components of the baseline error relative to the LOS are then derived, and their height error contribution is calculated.

\section{Simulations For the DEM CAlibration}

This section shows some height error simulation results (cf. also [10]) that prove that DEM adjustments are essential to achieve the specified TanDEM-X DEM height accuracy. It also includes simplified adjustment examples, which give an impression on the potentials of the concept.

\section{A. Modeling of Systematic Height Errors}

The models of the systematic errors described in Sections III and IV have been implemented in simulation software, which calculates the height error for an arbitrary TanDEM-X bistatic SAR data acquisition (cf. Fig. 11).

The color bar close to the individual 2-D height error plots represents the range of height errors of each realization. As shown in Fig. 3 and verified by Fig. 11, the systematic baseline error is on the order of meters, whereas the systematic instrument error is on the order of decimeters. The baseline error plot (left column in Fig. 11) can be detected again under the noiselike error in the total error plot (right column in Fig. 11). In this way, it is verified that the baseline error is normally the main systematic contribution to the TanDEM-X DEM height error.

The total height error profiles were then fitted with several functions. A statistical analysis of these simulations over different acquisition scenarios provided important results.

1) The total systematic height errors can be mainly described by a function depending on the azimuth time and an almost constant slope in ground range.

2) The evolution of the height error in the azimuth direction depends mainly on the error behavior of the different contributions plus the effect of the $h_{\mathrm{amb}}$ changes. Regions with stable $h_{\mathrm{amb}}$ (like Fig. 11) have almost linear behavior. If the DT is acquired in latitudes with fast $h_{\mathrm{amb}}$ changes (e.g., $>10 \%$ variation within the DT length), the behavior is better fitted with a higher order polynomial.

3) The slope in ground range (tilt) can be described by a linear function that depends on the parallel baseline error and is on the order of $2-5 \mathrm{~mm} / \mathrm{km}$.

4) For long DTs, a small change in tilt slope can be identified (torsion of the DEM in azimuth). It is assumed that the torsion changes linearly in azimuth. 


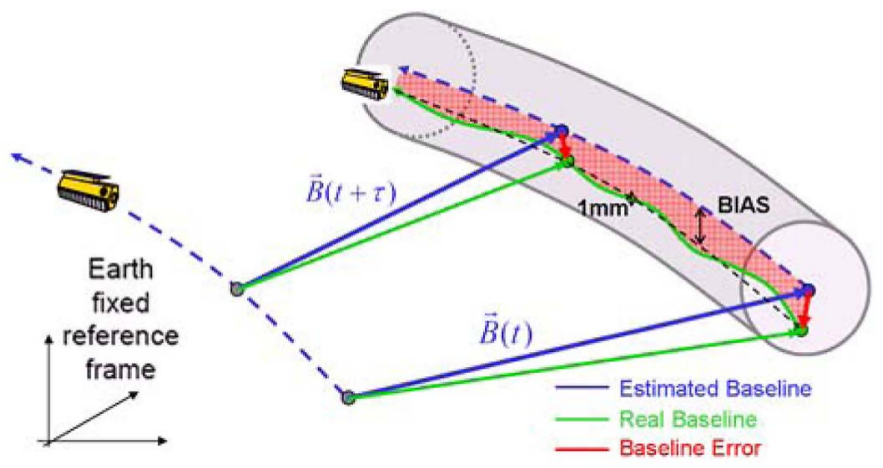

Fig. 9. TanDEM-X mission baseline error evolution over time.

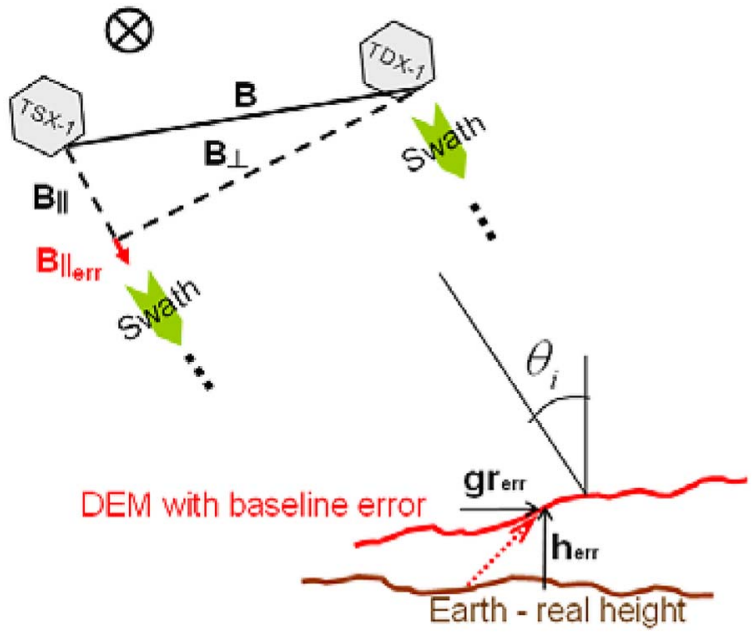

Fig. 10. Effects of a baseline LOS error on the DEM. Circle with a cross indicates flight direction into the image.

Therefore, the systematic height error realizations can be accurately fitted by a simple 2-D polynomial function. The ground range evolution can be approximated by linear functions and the one in azimuth by third-order polynomials (cf. also Fig. 12). The correction function is

$$
g(x, y)=a_{0}+a_{1} \cdot x+a_{2} \cdot x^{2}+a_{3} \cdot x^{3}+b_{1} \cdot y+k \cdot x \cdot y
$$

where

$g \quad$ height error correction function in $m$;

$x \quad$ position along the azimuth (flight) direction;

$y \quad$ position along the ground range (perpendicular to flight) direction;

$a_{0}, \ldots, a_{3}$ third-order polynomial coefficients that fit the azimuth error evolution;

$k$ (torsion) change in the ground range slope (fitted by a linear model) along the azimuth direction;

$b_{1} \quad$ slope in ground range.

As the raw DEMs delivered to the MCP are georeferenced, each of them has to be resampled into the corresponding $x, y$ DT relative coordinates, together with the available height references and the information from adjacent DTs, before deriving the coefficient values (8) with the least-squares method. After the 2-D height correction functions are applied, the DEMs are transformed back to the latitude-longitude grid.

The statistical analysis also allowed obtaining the typical values (mean and standard deviation) of the coefficients of (8),

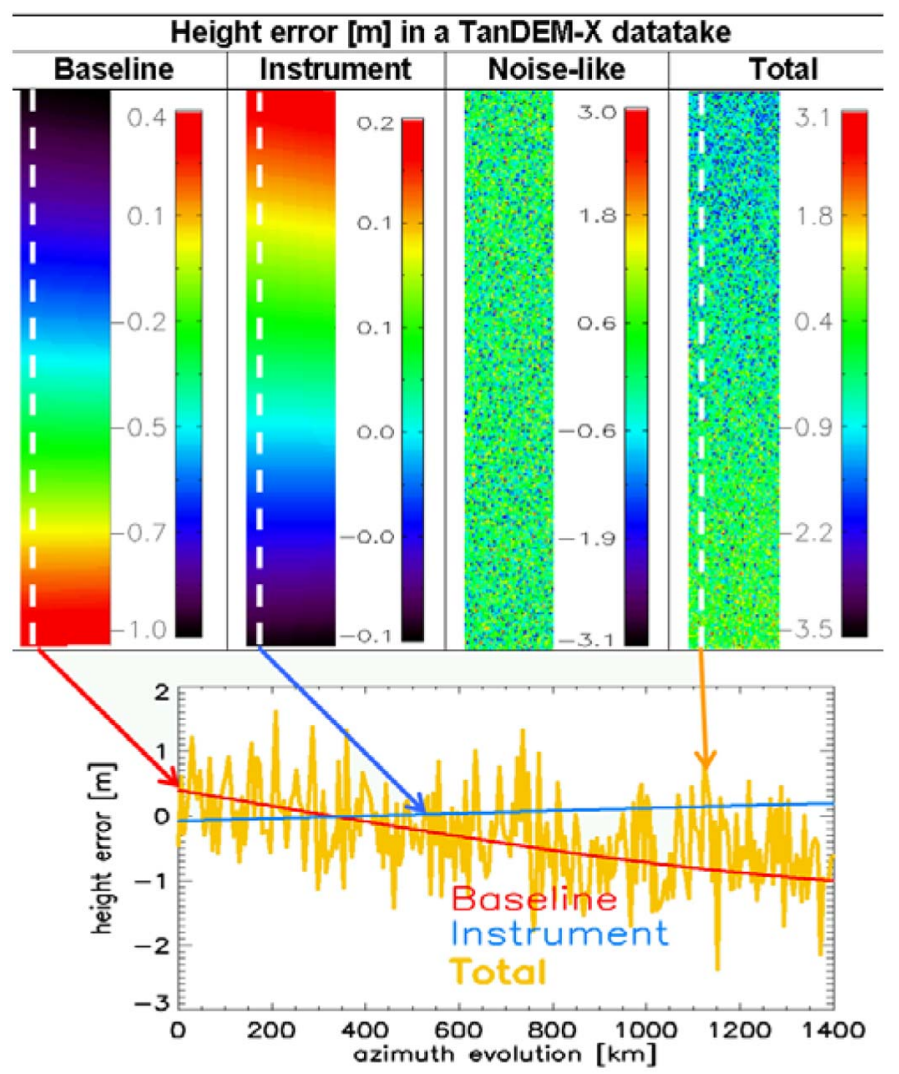

Fig. 11. (Upper row) Simulation of the height error contributions in meters of the individual sources (baseline systematic, instrument systematic, noiselike) and the combined total height error in a typical TanDEM-X mission DT. (Lower row) Height error evolution of the individual contributions above and the total error along the dashed white azimuth lines (constant ground range). DT location: $5^{\circ} \mathrm{N}$; ascending; DT length: $200 \mathrm{~s} / \sim 1400 \mathrm{~km}$; DT width: $\sim 30 \mathrm{~km}$; Swath $04 / \theta i \approx 38^{\circ}$; helix mission M-C1.

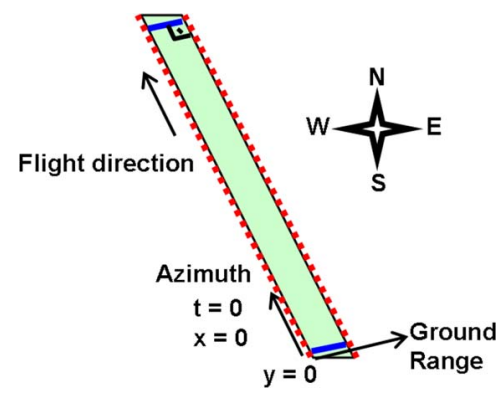

Fig. 12. Schematic of an interferometric TanDEM-X mission DT. The 2-D height error function is defined with respect to the axes $x$ (azimuth) and $y$ (ground range) with the origin in the beginning of the DT near range.

as listed in Table III. These values serve as reference for the $\mathrm{MCP}$ in order to check if the height error correction function reflects the expected height error behavior and to identify potential outliers. It has to be noted that in certain cases (regions with stable $h_{\mathrm{amb}}$ or DTs shorter than $700 \mathrm{~km}$ in azimuth), $a_{2}$ and $a_{3}$ can be set to zero as the azimuth evolution of the height error is almost linear.

\section{B. Adjustment Examples}

The goal of these simulations of the systematic height error (no noiselike components are considered here) is to validate the 
TABLE III

TyPiCAL VALUES OF THE COEFFICIENTS OF THE 2-D POLYNOMIAL MODELS FOR THE TANDEM-X HEIGHT ERRORS

\begin{tabular}{|l|l|l|l|l|l|l|}
\hline Coefficient & $\mathbf{a}_{\mathbf{0}}$ & $\mathbf{a}_{\mathbf{1}}$ & $\mathbf{a}_{\mathbf{2}}$ & $\mathbf{a}_{\mathbf{3}}$ & $\mathbf{b}_{1}$ & $\mathbf{k}$ \\
\hline Standard deviation & 2. & $3 . \mathrm{e}-4$ & $5 . \mathrm{e}-8$ & $8 . \mathrm{e}-12$ & $4 . \mathrm{e}-3$ & $1 . \mathrm{e}-6$ \\
\hline Mean value & 0 & 0 & 0 & 0 & 0 & 0 \\
\hline
\end{tabular}

2-D adjustment functions discussed in the previous section and support the development of the TanDEM-X MCP. This can be achieved if the relative height error in $100 \mathrm{~km} \times 100 \mathrm{~km}$ cells stays below $0.53 \mathrm{~m}$ (threshold derived in Section III-B) after the adjustment. It also helps to determine the required number, quality, and distribution of calibration references needed to successfully calibrate the global DEM.

Ground-control points (GCP) with a configurable accuracy can be introduced in arbitrary positions of the simulation region and provide absolute height references to the scene. All the GCPs selected in the simulations shown in this section are assigned an accuracy of $0.5 \mathrm{~m}(1 \sigma)$. In a real DEM adjustment, a set of GCPs are ideally available per DT.

In the first simulation example, a scenario containing five parallel ascending orbits with two acquisitions per strip as suggested by the acquisition plan (Section II-B), is adjusted (view Fig. 13).

The following simulation has the goal to test the performance of the adjustment with few high-quality GCPs. In this case, crossing orbits should be acquired at a certain distance from each other, crossing the maximum number of parallel DTs possible. Although the acquisition of crossing orbits is not foreseen in the acquisition plan (Section II-B), it could be applied as a back-up solution in the case of lack of height references in a certain region.

In this simulation, two GCPs are used in the crossing orbits, located at around $100 \mathrm{~km}$ from the group of adjacent DTs, as shown in Fig. 15. This compensates the along-track drift of the crossing orbit, which is the reference for the others. Another important conclusion of this simulation is the required distance between the crossing orbits in order to get a good error correction.

The calculated error realization and the results of its adjustment are shown in Figs. 13 and 14. One GCP is used for the adjustment of this scenario; however, more would be needed in a real case due to the mentioned random errors. The maximum relative error (in $100 \mathrm{~km} \times 100 \mathrm{~km}$ regions and shown in the lower part of the figures) has been improved from 2.0 to $0.44 \mathrm{~m}$, which keeps the height error within the requirements.

\section{Consequences for the DEM Calibration Concept}

The simulation results point out the need for applying a DEM calibration to the raw DEMs in order to achieve the mission accuracy requirements. Simulations were performed for different Earth regions and DT configurations, and show useful conclusions.

1) The final relative height accuracy is compliant with the defined DEM specification, assuming that the phase noise contributions do not exceed the specified limits, and the suggested accurate height references are available.
2) The final absolute height accuracy improves the $10-\mathrm{m}$ requirement of Table I and is on the order of the relative height accuracy.

3) In regions close to the equator or with dense vegetation, the GCP density strongly decreases compared with the rest of the landmass. In these cases, two long crossing orbits can help to obtain a stable adjustment net and a sufficient accuracy.

4) The parallel scenario has two major advantages.

a) The same strips are mapped in consecutive years during the same season. Hence, vegetation, tree cover, or ice cover are monitored in a similar state.

b) It simplifies the processing: the strips are acquired from the same orbit position.

The DEM calibration performance and the final DEM quality strongly depend on the precision of the applied height references. The simulations prove that relatively few highly accurate GCPs $(0.5 \mathrm{~m}-1 \sigma)$ are needed to achieve the desired height requirements. The TanDEM-X DEM calibration plan relies on height references provided by the Ice, Cloud, and land Elevation Satellite (ICESat) [17] and on the information provided by overlapping DTs.

Concerning ICESat altimetry data, selection criteria have been developed in order to filter the samples and keep the ones with best quality [17]. These are usually narrow altimeter pulse returns reflected in flat areas, and their assessed accuracy reaches $\sim 0.4 \mathrm{~m}(1 \sigma)$. The global coverage of the selected ICESat points has been also calculated, showing a sufficient density. Almost all the planned DT acquisitions are covered by several high-quality ICESat data, even in critical regions like rainforests and equatorial zones. This allows averaging several ICESat references. Additionally, as the posting of the TanDEM-X DEM is much finer than the ICESat-laser footprint, the heights have to be averaged with the appropriate weighting function before comparison. As most of the selected ICESat points lie on flat terrain, no accuracy loss is expected from the averaging process. The averaging helps to statistically reduce part of the random height error described in Section III-B.

Instead of using the whole overlapping regions for the adjustment, selected TanDEM-X DEM patches called tie points are used. They are strategically located along the planned overlapping regions and are regularly stored during the mission.

As already mentioned in Section II-C, the MCP is the operational tool for the TanDEM-X DEM calibration and mosaicking. The tool is run once the complete stack of raw DEMs of a certain region is available. As synthesized in the flowchart of Fig. 17, the algorithm derives, for each DT, the coefficients of the 2-D height correction function (8) by means of a leastsquares method with constraints, aided by the stored height references and tie points located within the interest region.

Fig. 16 shows the simulation results. The maximum relative error has improved from 2 to $0.38 \mathrm{~m}$, which meets the height requirement of $0.53 \mathrm{~m}$.

The derived height correction functions are applied to the corresponding DTs, thus obtaining calibrated and mosaicked DEMs. As the SAR images have been previously georeferenced (cf. Section II-C), and no considerable horizontal displacements of the DEMs are foreseen (as concluded in Section IV-D), just a height adjustment is needed to achieve the desired accuracy. 


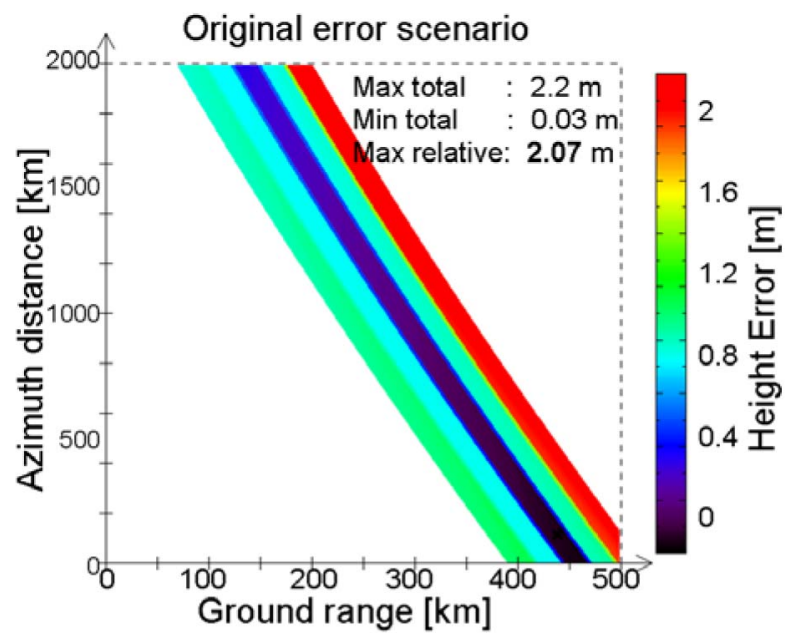

Fig. 13. Ten height error realizations (ascending adjacent DTs). Each of the five ground strips is acquired twice. Plot includes information about the maximum and minimum height error values within the scene and the worst relative height error in $100 \mathrm{~km} \times 100 \mathrm{~km}$ cells.

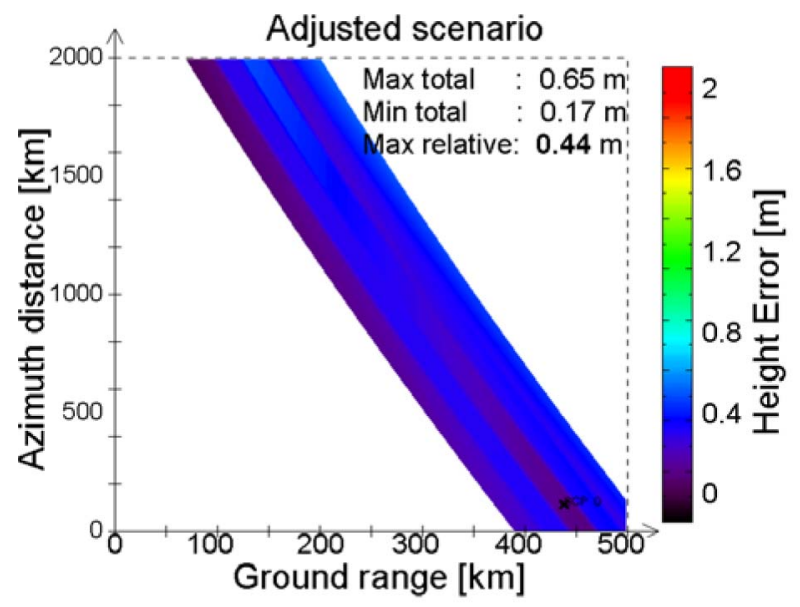

Fig. 14. Adjustment of the scenario. Plot includes information about the maximum and minimum height error values within the scene and the worst relative height error in $100 \mathrm{~km} \times 100 \mathrm{~km}$ cells.

\section{CONCLUSION}

This paper has described an exhaustive analysis of the residual-error behavior after bistatic instrument calibration. The efforts concentrate on correcting systematic errors originated mainly by baseline determination inaccuracies and residual instrument-phase drifts. Baseline error inaccuracies due to GPS errors are expected to be similar to the ones observed in missions like GRACE, which show a smooth baseline error evolution. On the other hand, onground tests provided information to describe the typical behavior of the systematic instrument-phase drift contributions. The combination of all these errors has been implemented in a software tool to simulate typical height error realizations for TanDEM-X mission DTs. The statistical analysis of these simulations provided a 2-D polynomial function useful for the DEM-height correction. The function coefficients are estimated for each DT by a leastsquares method within the MCP with the help of accurate height references like ICESat data and by using the information of the

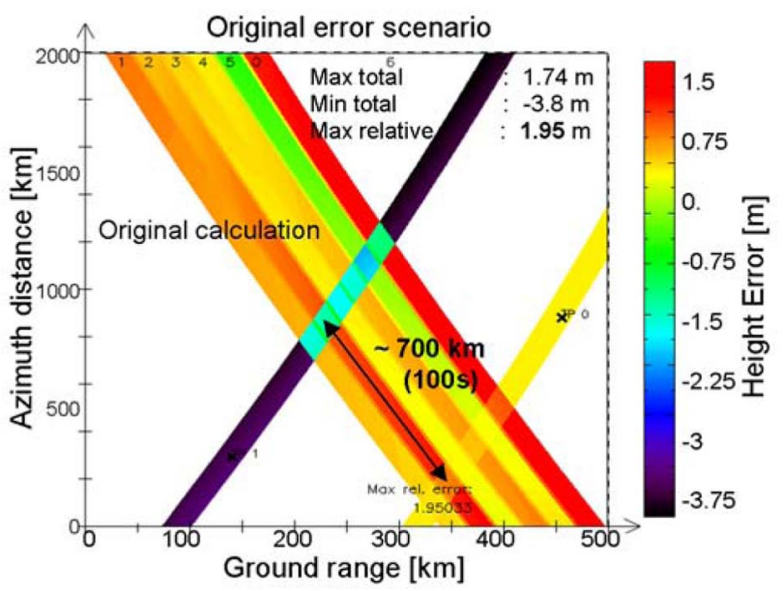

Fig. 15. Eight height error realizations (six ascending adjacent DTs and two crossing DTs at a distance of $700 \mathrm{~km}$ ). Plot includes maximum and minimum height error information within the scene and the worst relative height error in $100 \mathrm{~km} \times 100 \mathrm{~km}$ cells.

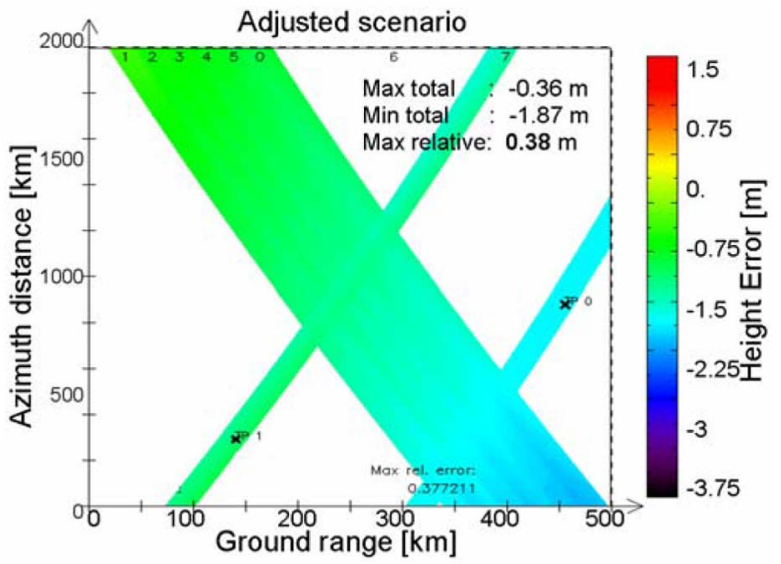

Fig. 16. Adjustment of the scenario. Plot includes maximum and minimum height error information within the scene and the worst relative height error in $100 \mathrm{~km} \times 100 \mathrm{~km}$ cells.

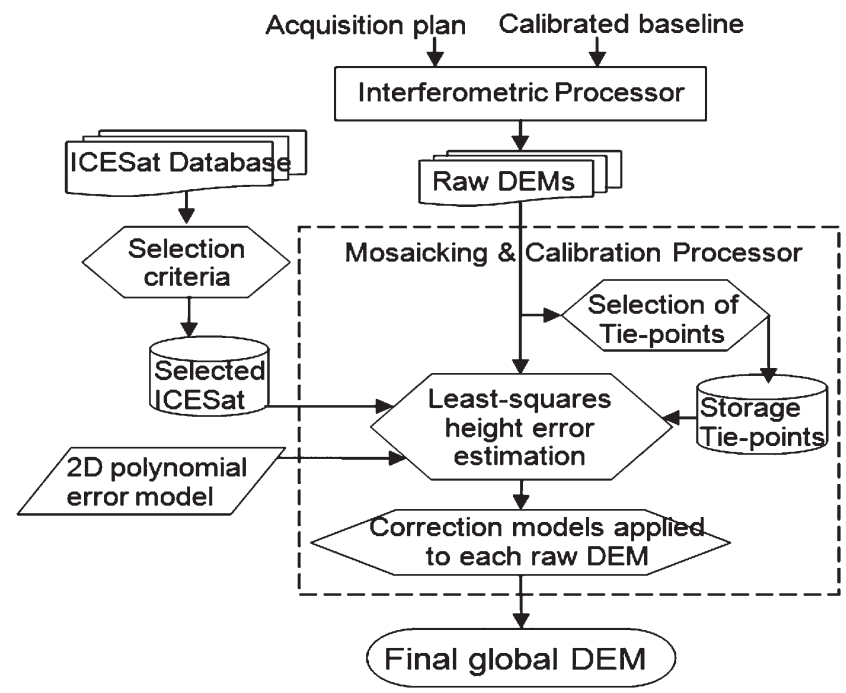

Fig. 17. Simplified flowchart of the DEM calibration concept. 
overlapping regions of adjacent DTs. The corresponding height correction 2-D functions need to be finally applied to obtain a calibrated global DEM that fulfils the demanding TanDEM-X DEM relative height requirements. The simulations show that the demanding relative height accuracy can be achieved through the DEM calibration process. At the same time, the simulations predict absolute height accuracies far better than the required $10 \mathrm{~m}$, assuming the expected availability and quality of height references.

\section{ACKNOWLEDGMENT}

The authors would like to thank the anonymous reviewers for their valuable comments and suggestions to improve this paper.

\section{REFERENCES}

[1] G. Krieger, A. Moreira, H. Fiedler, I. Hajnsek, M. Werner, M. Younis, and M. Zink, "TanDEM-X: A satellite formation for high-resolution SAR interferometry," IEEE Trans. Geosci. Remote Sens., vol. 45, no. 11, pp. 3317-3341, Nov. 2007.

[2] P. Rosen, S. Hensley, I. Joughin, F. Li, S. Madsen, E. Rodriguez, and R. Goldstein, "Synthetic aperture radar interferometry," Proc. IEEE, vol. 88, no. 3, pp. 333-382, Mar. 2000.

[3] NGA, MIL PRF 89048 HRTI-3-DEM Draft Document.

[4] B. Rabus, M. Eineder, A. Roth, and R. Bamler, "The Shuttle Radar Topography Mission (SRTM) - A new class of digital elevation models acquired by spaceborne radar," ISPRS J. Photogramm. Remote Sens., vol. 57, no. 4, pp. 241-262, Feb. 2003.

[5] D. Geudtner and M. Zink, "Interferometric calibration of the X-SAR system on the Shuttle Radar Topography Mission (SRTM/X-SAR)," in Proc. 21st Can. Remote Sens. Symp., Ottawa, ON, Canada, Jun. 1999, pp. 558-565.

[6] E. Rodriguez and J. M. Martin, "Theory and design of interferometric synthetic aperture radars," Proc. Inst. Elect. Eng. F-Radar Signal Process., vol. 139, no. 2, pp. 147-159, Apr. 1992.

[7] M. Lachaise, T. Fritz, and M. Eineder, "A new dual baseline phase unwrapping algorithm for the TanDEM-X mission," in Proc. EUSAR, Friedrichshafen, Germany, Jun. 2008.

[8] R. Bamler and M. Eineder, "Accuracy of differential shift estimation by correlation and split-bandwidth interferometry for wideband and delta-k SAR systems," IEEE Geosci. Remote Sens. Lett., vol. 2, no. 2, pp. $152-$ 155, Apr. 2005.

[9] B. Wessel, A. Gruber, J. Hueso Gonzalez, M. Bachmann, and A. Wendleder, "TanDEM-X: DEM calibration concept," in Proc. IEEE IGARSS, Boston, MA, Jul. 2008, pp. III-111-III-114.

[10] J. Hueso González, M. Bachmann, H. Fiedler, S. Huber, G. Krieger, M. Zink, and B. Wessel, "Development of TanDEM-X DEM calibration concept," in Proc. EURad, Munich, Germany, Oct. 2007, pp. 1743-1746.

[11] G. Krieger and M. Younis, "Impact of oscillator noise in bistatic and multistatic SAR," IEEE Geosci. Remote Sens. Lett., vol. 3, no. 3, pp. 424428, Jul. 2006

[12] M. Younis, R. Metzig, G. Krieger, and R. Klein, "Performance prediction and verification for bistatic SAR synchronization link," in Proc. EUSAR, Dresden, Germany, May 2006.

[13] S. Huber, M. Younis, and G. Krieger, "TanDEM-X performance analysis," in Proc. EUSAR, Friedrichshafen, Germany, Jun. 2008.

[14] O. Montenbruck, L. v. Barneveld, Y. Yoon, and P. Visser, "GPSbased precision baseline reconstruction for the TanDEM-X SARformation," in Proc. 20th Int. Symp. Space Flight Dyn., Annapolis, MD, Sep. 2007.

[15] R. Kroes, O. Montenbruck, W. Bertiger, and P. Visser, "Precise GRACE baseline determination using GPS," GPS Solut., vol. 9, no. 1, pp. 21-31, Apr. 2005.

[16] M. Schwerdt, B. Bräutigam, M. Bachmann, B. Döring, D. Schrank, and J. Hueso González, "TerraSAR-X calibration results," in Proc. IEEE IGARSS, Boston, MA, Jul. 2008, pp. II-205-II-208.

[17] J. Hueso González, M. Bachmann, R. Scheiber, and G. Krieger, "TanDEM-X DEM calibration and processing experiments with E-SAR," in Proc. IEEE IGARSS, Boston, MA, Jul. 2008, pp. III-115-III-118.

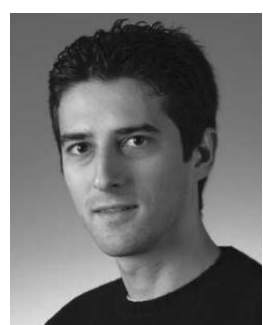

Jaime Hueso González received the M.S. degree in telecommunication engineering from the Polytechnic University of Valencia, Valencia, Spain, in 2003.

From 2004 to 2005, he was a Microwave Engineer with the European Space Agency, Noordwijk, The Netherlands, where he researched on microwavefilter design, high-power testing, and multipactor effects on spaceborne microwave waveguides. Since 2006, he has been with the German Aerospace Center (DLR), Wessling, Germany, where he specializes on satellite SAR technology. He has been working as a Calibration Engineer for projects like TerraSAR-X, TanDEM-X, and GMES Sentinel-1. His major research interests include the development of innovative calibration concepts for digital elevation models and satellite baseline determination.

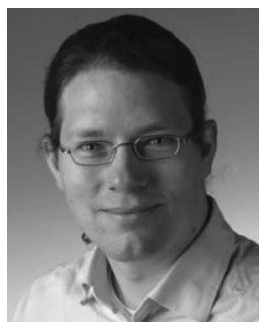

Markus Bachmann received the Dipl.-Ing. degree in electrical engineering from the Technical University of Karlsruhe, Karlsruhe, Germany, in 2005.

Since 2005, he has been with the Microwaves and Radar Institute, German Aerospace Center (DLR), Wessling, Germany, where he is currently a Calibration Engineer in SAR calibration. In the TerraSAR-X project, he is responsible for the Antenna Model calibration; in TanDEM-X, for the DEM calibration concept.

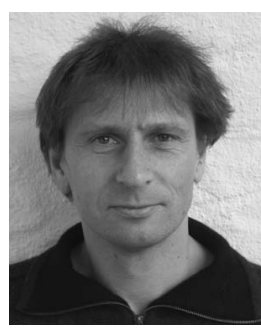

Gerhard Krieger (A'04-M'04) received the Dipl.Ing. (M.S.) and Dr.-Ing. (Ph.D.) degrees (with honors) in electrical and communication engineering from the Technical University of Munich, Munich, Germany, in 1992 and 1999, respectively.

From 1992 to 1999 , he was with the LudwigMaximilians University, Munich, where he conducted multidisciplinary research on neuronal modeling and nonlinear information processing in biological and technical vision systems. Since 1999, he has been with the Microwaves and Radar Institute, German Aerospace Center (DLR), Wessling, Germany, where he developed signal and image processing algorithms for a novel forward looking radar system employing the principle of digital beamforming on receive. From 2001 to 2007, he led the New SAR Missions Group which pioneered the development of advanced bistatic and multistatic radar systems as exemplified by the forthcoming TanDEM-X mission as well as innovative multichannel SAR techniques and algorithms for high-resolution wide-swath SAR imaging. Since 2008, he has been Head of the new Radar Concepts Department. His current research interests focus on the development of multichannel radar techniques and algorithms for innovative MIMO SAR systems, the demonstration of novel interferometric and tomographic Earth-observation applications, and the conceptual design of advanced bi- and multistatic radar missions.

Dr. Krieger is the recipient of several national and international awards, including the IEEE Transactions Prize Paper Award of the Geoscience and Remote Sensing Society and the Otto Lilienthal Sabbatical of the German Aerospace Center, DLR.

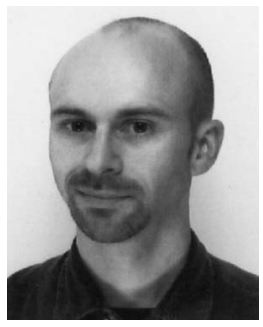

Hauke Fiedler received the Diploma degree in physics and the Dr. degree in astronomy from the Ludwig-Maximilians-Universität of Munich, Munich, Germany, in 1994 and 2000, respectively, and the master degree of Space Systems Engineering of the Delft University of Technology (TU Delft), Delft, The Netherlands, in 2009.

From 1994, he was in the cataclysmic binary group with the Institute of Astronomy and Astrophysics with his work focused on Hard and Soft X-ray Interacting Binaries, Echo-Tomography, accretion disks and related subjects. Since 2001, he has been with the Microwaves and Radar Institute, German Aerospace Center (DLR), Wessling, Germany, where he is working on future satellite missions for remote sensing with synthetic aperture radar for the Earth and the Moon, particularly on formation and configuration concepts, bi- and multistatic SAR performance analyses, and satellite mechanics. He is involved in the TerraSAR-X project and particularly in the TanDEM-X project. 